\title{
Identifying culturally appropriate strategies for coronary heart disease secondary prevention in a regional Aboriginal Medical Service
}

\author{
Dhruv Govil ${ }^{\mathrm{A}}$, Ivan Lin ${ }^{\mathrm{B}, \mathrm{C}}$, Tony Dodd ${ }^{\mathrm{C}}$, Rhonda Cox ${ }^{\mathrm{B}}$, Penny Moss ${ }^{\mathrm{A}}$, \\ Sandra Thompson ${ }^{\mathrm{B}}$ and Andrew Maiorana ${ }^{\mathrm{A}, \mathrm{D}, \mathrm{E}}$ \\ ${ }^{A}$ School of Physiotherapy and Curtin Health Innovation Research Institute, Curtin University, \\ GPO Box U1987, Perth, WA 6845, Australia. \\ ${ }^{B}$ Combined Universities Centre for Rural Health, University of Western Australia, PO Box 109 Geraldton, \\ WA 6531, Australia. \\ ${ }^{\mathrm{C}}$ Geraldton Regional Aboriginal Medical Service, Rifle Range Road, Rangeway, WA 6530, Australia. \\ DAdvanced Heart Failure and Cardiac Transplant Service, Royal Perth Hospital, Box X2213 GPO, \\ Perth, WA 6847, Australia. \\ ${ }^{E}$ Corresponding author. Email: A.Maiorana@curtin.edu.au
}

\begin{abstract}
Aboriginal Australians experience high rates of coronary heart disease (CHD) at an early age, highlighting the importance of effective secondary prevention. This study employed a two-stage process to evaluate CHD management in a regional Aboriginal Medical Service. Stage 1 involved an audit of 94 medical records of clients with documented CHD using the Audit and Best Practice in Chronic Disease approach to health service quality improvement. Results from the audit informed themes for focus group discussions with Aboriginal Medical Service clients $(n=6)$ and staff $(n=6)$ to ascertain barriers and facilitators to CHD management. The audit identified that chronic disease management was the focus of appointments more frequently than in national data $(P<0.05)$, with brief interventions for lifestyle modification occurring at similar or greater frequency. However, referrals to follow-up support services for secondary prevention were lower $(P<0.05)$. Focus groups identified psychosocial factors, systemic shortcomings, suboptimal medication use and variable awareness of CHD signs and symptoms as barriers to CHD management, whereas family support and culturally appropriate education promoted health care. To optimise CHD secondary prevention for Aboriginal people, health services require adequate resources to achieve best-practice systems of follow up. Routinely engaging clients is required to ensure services meet diverse community needs.
\end{abstract}

Additional keywords: cardiac rehabilitation, cardiovascular disease, cultural security, medication, risk factors.

Received 14 September 2012, accepted 23 April 2013, published online 12 June 2013

\section{Introduction}

Coronary heart disease (CHD) is the leading cause of morbidity and mortality in Aboriginal Australians, occurring at higher rates and a younger age than in other Australians (Katzenellenbogen et al. 2010). While the factors underlying this disparity are complex, the barriers Aboriginal people face in accessing specialised health services is a significant contributing factor (Cunningham et al. 2005). Improving equity in cardiovascular health management between Aboriginal and non-Aboriginal Australians is an urgent priority in Australian health care in order to address the well-documented life expectancy gap between the two groups (Vos et al. 2009).

An Aboriginal Community Controlled Health Service, also known as an Aboriginal Medical Service (AMS), is a primary health care service initiated and operated by the local Aboriginal community to deliver holistic, comprehensive and culturally appropriate health care. Its management is overseen by a locally elected Board of Management. The development of AMSs acknowledges that general health services often do not efficiently meet the needs of Aboriginal people, and that health services for Aboriginal people can be more effective when delivered by health practitioners with an understanding of Aboriginal culture and lifestyles (Couzos and Murray 2003). Aboriginal Medical Services are the preferred medical provider for many Aboriginal people because they offer health care that is sensitive to the needs, views, values and expectations of Aboriginal people (Eckerman et al. 2010). In rural and remote locations, AMSs may be the sole provider of CHD management through the provision of medical review, medication surveillance and support for risk factor modification. 


\section{What is known about the topic?}

- Aboriginal people experience coronary heart disease at an earlier age than the non-Aboriginal population. However, specialised cardiovascular health services are often not available to Aboriginal people living in regional and remote communities, and where they exist, are not accessed because of marginalisation and real or perceived discrimination.

\section{What does this paper add?}

- This paper identifies the cultural, socioeconomic and service-related challenges of managing cardiovascular care in a regional Aboriginal Medical Service and highlights the importance of community consultation in ensuring services are culturally appropriate.

The aim of this study was to examine CHD services and practices within an AMS in Western Australia (WA) and to use these outcomes to inform discussion with staff and clients about strengthening cardiovascular health management.

\section{Methods}

\section{Study design}

The study employed complementary quantitative and qualitative methods. Stage One involved a quality improvement audit of CHD health care. Data from this audit was reviewed and formed the basis for interview questions and focus group discussions in Stage Two. Mixed methods provide a methodologically rigorous approach to health service research that has been found to generate deeper insights than quantitative or qualitative methods alone (Bryman 1995; Moffatt et al. 2006) and embraces the tenets of Aboriginal health research (NHMRC 2010).

\section{Stage one: quality improvement audit \\ Audit sample}

All clients in the AMS's database who met the following criteria were included: (1) documented diagnosis of CHD (including angina, ischaemic heart disease and myocardial infarction); (2) Aboriginal and/or Torres Strait Islander; (3) aged 18 years or older.

\section{Assessment}

The audit was conducted using the Audit and Best Practice in Chronic Disease (ABCD) methodology for reviewing the medical management of chronic conditions in Aboriginal people (Bailie et al. 2007). Data were gathered on clinical service efficiency relative to recognised best practice guidelines. Deidentified clinical records meeting the inclusion criteria were audited and compared with national data derived from 11 different centres across Australia, which had completed CHD audits in the same quarter as the audit date of the current project (11 November 2010) or in the three previous quarters, to make up a 12-month period (One21 seventy 2011).
Stage two: focus group discussions/interviews

\section{Consultation phase}

Visits to the AMS by the researchers and involvement of local Aboriginal personnel in the research team established trust and an understanding of the AMS's operations. In addition, involvement of Aboriginal health care professionals and Elders from the Aboriginal community in formulating the focus group discussion topics ensured the research process was undertaken in a culturally appropriate manner (NHMRC 2003), and together with feedback to a wider group of staff familiar and committed to quality improvement, ensured the research was participatory. This type of 'ecological community approach' has been recognised globally in a recent systematic review of successful cardiovascular health programmes in Indigenous communities (Huffman and Galloway 2010).

\section{Implementation phase}

Two focus groups were recruited following the consultation phase. Group One (staff) comprised two Aboriginal Health Workers (AHWs) and one GP, nurse, allied health practitioner and administrator who were involved in the management and delivery of CHD-related services. The second focus group comprised six local Aboriginal community members (three male and three female). Flyers were distributed around the AMS calling for volunteers from clients with a history of CHD. Nine clients initially accepted the invitation but three withdrew due to competing commitments.

Where elaboration was sought on focus group discussion points, follow-up one-on-one interviews were conducted.

\section{Focus group structure}

All focus groups and interviews were conducted by the same investigator in collaboration with an AHW to ensure cultural security. The interviews employed open-ended questions to facilitate in-depth discussions of the experiences and perceptions of cardiac services by Aboriginal people.

\section{Data analysis}

The audit was performed by researchers experienced with the electronic patient information record system (Communicare) and the audit data collection form. Automated reports were generated providing simple descriptive analyses that compared audit results against national figures. A chi-square contingency table was used to determine the level of significance $(P \leq 0.05)$ between the AMS and national data.

Discussions and interviews were audio-recorded, transcribed and imported into NVivo7 software (Version 9, QSR International Pty Ltd, Melbourne, Vic., Australia). A framework analysis was employed, which involved reviewing the transcripts against the framework from the audit to identify recurring themes reflecting common views and experiences. These were collated and supported by de-identified quotes from participants.

\section{Ethics}

Ethical approval was obtained from the Western Australian Aboriginal Health Information and Ethics Committee (313 10/ 10), the Aboriginal Medical Service and the Curtin University Human Research Ethics Committee (HR 124/2010). All 
participants involved in discussions provided written informed consent.

\section{Results}

\section{Stage one: quality improvement audit}

The audit in the current study reviewed all records of clients within the AMS who were identified with CHD. In comparison, the national comparator data comprised samples of 30 records from several centres, stratified by age and gender. However, neither age nor gender was significantly different between the audit and national data.

The AMS in the current study had a higher proportion of clients who attended for chronic disease management at their last appointment, but there were no other significant differences between the study cohort and the national dataset (Table 1).

Coronary heart disease risk factors documented in the medical records of the audit group were compared with national data (Table 2). The incidence of hypertension and hyperlipidaemia were lower and low-risk alcohol use was higher in the audit group, but there were no other differences.

Brief interventions and discussions about lifestyle to address CHD risk factors occurred at least as frequently as reported in the national data (Table 3). However, formal chronic disease management plans and client referral to support services for

Table 1. Key characteristics of clients included in the audit AMS, Aboriginal Medical Service; CDM, chronic disease management

\begin{tabular}{lccc}
\hline Profile of clients & $\begin{array}{c}\text { AMS } \\
(n=94)\end{array}$ & $\begin{array}{c}\text { National } \\
(n=234)\end{array}$ & $P$-value \\
\hline Aged 45-64 years & $68 \%$ & $62 \%$ & 0.29 \\
Male gender & $64 \%$ & $52 \%$ & 0.05 \\
Attendance at AMS in past 6 months & $83 \%$ & $90 \%$ & 0.07 \\
Last attendance at AMS for CDM & $81 \%$ & $61 \%$ & 0.001 \\
\hline
\end{tabular}

Table 2. Coronary heart disease risk-factor characteristics of clients obtained from medical records

\begin{tabular}{|c|c|c|c|}
\hline Risk factor & AMS & National & $P$-value \\
\hline Current smoker & $31 \%$ & $37 \%$ & 0.28 \\
\hline \multicolumn{4}{|l|}{ Body mass index } \\
\hline$\geq 25-\leq 29.9$ & $29 \%$ & $21 \%$ & 0.13 \\
\hline$\geq 30$ & $37 \%$ & $39 \%$ & 0.78 \\
\hline Blood pressure $>130 / 80^{\mathrm{C}}$ & $45 \%$ & $59 \%$ & 0.02 \\
\hline \multicolumn{4}{|l|}{ Alcohol use } \\
\hline Higher risk $^{\mathrm{A}}$ & $5 \%$ & $11 \%$ & 0.10 \\
\hline Low risk $^{\mathrm{B}}$ & $38 \%$ & $20 \%$ & 0.001 \\
\hline \multicolumn{4}{|l|}{ Cholesterol $^{\mathrm{C}}$} \\
\hline$\geq 4.0$ and $\leq 5.5 \mathrm{mmol} / \mathrm{L}$ & $51 \%$ & $45 \%$ & 0.31 \\
\hline$>5.5 \mathrm{mmol} / \mathrm{L}$ & $12 \%$ & $12 \%$ & 0.95 \\
\hline \multicolumn{4}{|l|}{ Comorbidities } \\
\hline Type II diabetes & $59 \%$ & $59 \%$ & 0.94 \\
\hline Hypertension & $48 \%$ & $65 \%$ & 0.004 \\
\hline Hyperlipidaemia & $32 \%$ & $51 \%$ & 0.002 \\
\hline
\end{tabular}

${ }^{A}>$ Two standard drinks per day on average.

${ }^{\mathrm{B}} \leq$ Two standard drinks per day on average.

${ }^{\mathrm{C}}$ Most recent entry on client's medical record. targeted management of risk factors occurred less often. The proportion of patients prescribed aspirin, long-acting nitrates and lipid-lowering drugs was lower in the study cohort but there were no differences for other medications.

\section{Stage two: focus group discussions/interviews}

As a result of the audit findings, participants were asked to comment on: use of the AMS for CHD care and adherence to $\mathrm{CHD}$ management plans; knowledge of CHD risk factors; lifestyle modifications for CHD management; medication use; and suggestions for how the AMS could better service the local community.

Nine common themes related to CHD management emerged from the focus groups. Quotes from participants are provided to support each theme.

(1) Preference for receiving $C H D$ health care at a local AMS

Clients reported a strong preference for attending the local AMS for health management (opposed to a mainstream service in the nearest large city, Perth), citing greater trust in the AMS staff, the availability of multiple services, confidence in staff expertise and proximity to home and family support.

\section{It's a good place to sit around and talk about your health.} You feel comfortable.

I always feel scared when I have to leave my home here and go away. You know that you belong here and itfeels strange not being in your home.

Table 3. Secondary prevention practices ACE, angiotensin-converting enzyme; AMS, Aboriginal Medical Service

\begin{tabular}{lrrr}
\hline Service delivered in past 12 months & AMS & National $^{\mathrm{A}}$ & $P$-value \\
\hline Current Chronic Disease Management Plan & $14 \%$ & $35 \%$ & $<0.001$ \\
Brief intervention for risky alcohol use & $100 \%$ & $82 \%$ & $<0.001$ \\
Referral to risky alcohol use programme & $0 \%$ & $14 \%$ & $<0.001$ \\
For clients with body mass index $\geq 25$ & & & \\
$\quad$ Brief intervention for obesity & $58 \%$ & $59 \%$ & 0.94 \\
$\quad$ Referral to obesity management services & $31 \%$ & $40 \%$ & 0.11 \\
Scheduled services delivered & & & \\
Blood pressure (within past 6 months) & $77 \%$ & $84 \%$ & 0.11 \\
Weight (within 6 months) & $76 \%$ & $71 \%$ & 0.40 \\
Waist circumference (within 6 months) & $32 \%$ & $41 \%$ & 0.12 \\
Factors discussed in past 12 months & & & \\
Nutrition & $71 \%$ & $64 \%$ & 0.21 \\
Physical activity & $73 \%$ & $62 \%$ & 0.05 \\
Chronic disease management & $85 \%$ & $80 \%$ & 0.10 \\
Prescribed medications & & & \\
ACE inhibitors & $41 \%$ & $49 \%$ & 0.21 \\
Angiotensin 2 receptor blockers & $21 \%$ & $16 \%$ & 0.24 \\
Anti-hypertensive drugs & $36 \%$ & $38 \%$ & 0.75 \\
Lipid-lowering drugs & $47 \%$ & $63 \%$ & 0.008 \\
Aspirin or other anti-clotting drug & $52 \%$ & $67 \%$ & 0.01 \\
Beta blockers & $22 \%$ & $33 \%$ & 0.06 \\
Nitrates: short acting & $13 \%$ & $17 \%$ & 0.33 \\
Nitrates: long acting & $2 \%$ & $10 \%$ & 0.02 \\
\hline One2lseventy (2011). & & \\
\hline
\end{tabular}

${ }^{\mathbf{A}}$ One21seventy (2011). 
Staff also highlighted the importance of trust in the AMS:

[The AMS] is close, local and people know who's here. I guess some people may see it as a safer place than going to Perth.

(2) Social factors may be a barrier to attending the AMS

Interviewees identified competing priorities that prevented regular attendance at the AMS, including family and work commitments:

Some people ... have so many problems in their life, why just fix one when there's still all the other ones hanging on their head?

This was especially relevant for regular attendance at a programme for chronic disease management:

It's hard as there's cooking and cleaning to do and the kids to chase after.

Staff identified similar reasons for non-attendance:

Transport, grandkids and other family responsibilities.

Staff also indicated that clients' concerns about confidentiality were a potential barrier to attendance:

.. because they have relatives working here. They won't tell the health worker about their drinking, as they want to avoid shame.

\section{(3) Family support can enhance health knowledge}

Clients identified the value of education for behaviour change in a supportive family environment:

Education is important ... you gotta understand what's going on inside your body to be able to fix it, and sometimes you need your family to explain it.

(4) Knowledge of heart disease and related signs/ symptoms were variable

Some clients demonstrated limited knowledge of the clinical manifestations of CHD:

I never bothered with [the symptoms]... I just never thought it was important and then it happens and you end up in a lot of strife.

However, awareness of CHD risk factors may increase after a cardiac event:

I know now high blood pressure can cause problems, so I watch that closely since my heart attack.

Staff often had difficulty conveying the seriousness of ischaemic symptoms to clients:

We could go into more detail but people just tune out ... it's difficult to explain the seriousness of [ischaemic symptoms].

\section{(5) Suboptimal risk factor modification was common}

Some clients reported difficulty modifying their lifestyles. However, practical interventions initiated by staff were helpful:
I've used the diet diary they gave me, I didn't write in it much but having it on the table sort of reminded you a little to stick to the plan.

(6) Inconsistent medication adherence.

Clients reported fluctuating levels of adherence with prescribed medication:

I think it's a few pills each fortnight. Just depends on how I' $m$ feeling or what the doc says. I' $m$ pretty good with taking it after I've seen the doctor, but yeah, when I' $m$ feeling good, not so much.

\section{(7) Appropriate education strategies}

Clients identified the importance of understandable health education:

If you can't understand what [the doctors] are saying then why bother listening?

Demonstration and experiential learning, rather than purely didactic instruction alone, was favoured by clients:

...you need to be shown as well, 'cos we all like to experience things. It's just boring otherwise.

Staff felt that educational messages needed to be positive and consistent to be effective:

We need to talk consistently about the benefits of healthy life rather than all the negatives.

(8) Systemic shortcomings may hinder effective health management

Clients reported that extended waiting times for appointments can cause dissatisfaction:

The biggest problem is the waiting time ... [it] may be one of the things that turns men off. The women will wait and chat to each other.

Inadequate referral and information about follow-up services from mainstream tertiary health care also impacted on selfmanagement of CHD:

They didn't say anything at the time about what to do after I left hospital or who to see or when I needed seeing. The hospital said they'd call. I' $m$ still waiting.

Staff acknowledged systemic issues, highlighting deficiencies in the following areas:

(i) Limited capacity within the AMS:

...you've also got to look at capacity and capabilities. Doctors are on the limit, you need to think about the whole system and make sure there's the resources.

(ii) The need for ongoing professional development, employing culturally appropriate teaching methods:

People need to understand their roles and the roles of those around them. Aboriginal Health Workers aren't very good in a lecture environment, they are more tactile, practical and visual learners. 


\section{(9) Support for cardiac rehabilitation}

Clients and staff indicated strong support for cardiac rehabilitation conducted by the AMS:

$A$ heart clinic and rehabilitation programme would be fantastic ... to have an Aboriginal organisation fixing it would be great.

\section{Discussion}

This audit of clients with CHD at a regional AMS identified that, while secondary prevention practices were frequently discussed, there was a relatively low utilisation of chronic disease management plans or referral to support services for risk factor modification. Subsequent focus group discussions identified a variety of barriers to $\mathrm{CHD}$ care, highlighting the complex nature of managing CHD in Aboriginal people. The findings suggest that providing culturally appropriate and accessible secondary prevention services requires additional resources to reduce the burden of Aboriginal CHD in regional Australia, but that AMSs are well placed to contribute.

Formalised care plans have been shown to reduce reactive care to treat acute exacerbations of chronic conditions (Østbye et al. 2005). Despite $81 \%$ of clients attending the AMS for chronic disease management in the 12 months preceding the audit, only $14 \%$ had a formal chronic disease management plan. Focus groups revealed several possible reasons for this discrepancy. For on-site programmes, limited staff resources, clients' competing commitments and transport difficulties may have discouraged staff from initiating plans. Given the strong preference expressed by focus groups for AMS-based services that offer health care in a culturally appropriate manner, there may have been reluctance to establish a care plan that relied on external mainstream services. Furthermore, the cost associated with accessing allied health services may be prohibitive for some Aboriginal people, consistent with observations in the non-Indigenous population (Schofield 1999). Accordingly, brief interventions were more commonly used to address nutrition/obesity, physical activity and risky alcohol use.

Underutilisation of care plans is not exclusive to AMSs; a recent report by a non-Indigenous, rural GP service reported that use of care plans was well below national benchmarks (Pierce 2009). This study found that training in the appropriate use of enhanced primary care items, software to support utilisation and promotion of practice nurse participation increased the use of enhanced primary care plans. These strategies might also work well in an AMS, as might Medicare-subsidised allied health services (Foster et al. 2008).

The barriers to accessing CHD health care described by clients in the current study are consistent with those observed elsewhere (Shepherd et al. 2003; DiGiacomo et al. 2010b). Inadequate service linkage between tertiary and primary health services was a problem reported by both clients and staff of the AMS. Systemic problems have been persistent in Aboriginal health care due to inconsistent and insufficient data collection processes, poor communication between health providers and multiple, incompatible information systems and technologies (Shepherd et al. 2003; DiGiacomo et al. 2010a). More effective systems of communication and follow up between the various levels of health care are required.
While Australia has universal health insurance for Australian citizens and permanent residents, the Australian Government has recognised that the large discrepancies in health outcomes and life expectancy between Aboriginal and non-Aboriginal people require more proactive measures to improve health care access and delivery to Aboriginal people. Therefore, special mechanisms have been introduced to encourage best practice care for Aboriginal people and assist Aboriginal people to have access to prescribed medications. In general, while these initiatives have been welcomed, challenges remain in their implementation to achieve high uptake and quality of care. (Stoneman and Taylor 2007; Halcomb et al. 2010) In the current study, prescribed cardiac medications recorded in clients' medical records were lower than has been reported in primary care for the overall Australian population (Heeley et al. 2010), consistent with previous findings for Aboriginal people (AIHW 2010). Ongoing efforts are required to address this disparity and to improve evidence-based medical therapy for Aboriginal people, with the relationship between the patient and prescribing doctor critical in this respect (Thompson and Walker 2011).

The focus groups revealed broad support for a dedicated cardiac rehabilitation programme within the AMS. Cardiac rehabilitation services are multidisciplinary, providing education and guidance for individualised secondary prevention and client follow up (Redfern et al. 2011). Such programmes are well suited to primary care, because they complement the role of the GP, who may have insufficient time to address chronic disease management comprehensively during a standard consult. Aboriginal Health Workers can be invaluable in this regard, providing education and support in a culturally secure environment. When AMS-based programmes are conducted in collaboration with experienced mainstream health professionals, an environment for knowledge exchange and experiential learning is established (Dimer et al. 2013). In the current study, AMS staff identified that they had difficulty conveying some essential cardiovascular health messages to clients, highlighting the importance of educational support and training.

The focus group discussions in the current study revealed several important considerations to help improve CHD management for Aboriginal people. Programmes should deliver health information consistent with preferred Aboriginal ways of learning, in an interactive rather than didactic fashion and avoiding negative stereotypes. They should be inclusive of family, recognising the important role family members have in helping manage an individual's health, with family included in lifestyle management discussions at the discretion of clients. A range of solutions should be considered to improve access and uptake, including transport assistance, flexible programme times and modes of delivery (including after-hours, home visits) and crèche facilities for clients with children.

Findings from this study have important implications for primary care. Traditional doctor-based education may not effectively engage Aboriginal clients; even after diagnosis with CHD, some participants did not have a good understanding of the disease and its consequences, so were not proactive in managing their conditions. Alternative approaches to educating patients need to be tried and evaluated. Without patients developing an understanding of what CHD is and its implications for their health, it may be difficult to improve adherence to evidence-based 
management. While the focus of this research was CHD, these observations are also likely to be relevant to the management of other chronic conditions in primary care, such as diabetes and renal disease, which share many common risk factors, and in combination with CHD, account for $\sim 50 \%$ of the life-expectancy gap (Australian Bureau of Statistics and Australian Institute of Health and Welfare 2008). Finally, the methodological approach taken in this study may also be effective for evaluating health care services in other marginalised groups, including a low socioeconomic demographic and where English is a second language.

\section{Limitations}

It is important to recognise that the service participating in this study, as well as those from which the national comparator data were drawn, are selected groups undertaking quality improvement approaches and therefore likely to have better patient follow up than health services not undertaking such processes. While the audit sample in the current study reviewed all records of clients within the AMS, the national comparator data comprised of samples of 30 records from several centres, stratified by age and gender. However, neither age nor gender was significantly different between the audit and national data. Importantly, documenting a complete profile of all clients with a recorded diagnosis of CHD provided a unique review of cardiovascular health management practices within an AMS and was necessary for informing the focus group discussions. The data related to medication use describes medication as prescribed and may overestimate actual usage.

The focus group cohort was recruited from a regional area of WA. While many findings are likely to be transferable, some may be community-specific. Although the study included a crosssection of clients, convenience sampling meant participants were engaged with the AMS and likely to have better health care than less engaged individuals and with opinions that may not necessarily represent those who are disenfranchised.

\section{Conclusion}

Aboriginal Medical Services are an important provider of CHD health care in regional Australia but may lack the resources to provide comprehensive secondary prevention. Establishing a structured cardiac rehabilitation programme within an AMS is likely to complement other medical services and strengthen follow-up care by supporting risk factor modification and medication compliance. Such programmes should be designed in consultation with the local community to ensure they meet their needs and expectations.

\section{Conflicts of interest}

None declared.

\section{Acknowledgements}

The authors acknowledge Professor Ross Bailie for his support of the project and advice regarding the ABCD audit process. We are indebted to the AMS that took part in the study and the staff and clients who took part in the focus groups. This study was funded by a Curtin University Internal Research Grant.

\section{References}

Australian Bureau of Statistics and Australian Institute of Health and Welfare (2008). 'The Health and Welfare of Australia's Aboriginal and Torres Strait Islander Peoples.' ABS Catalogue No. 4704.0. AIHW Catalogue No. IHW 21. (ABS and AIHW: Canberra)

Australian Institute of Health and Welfare (2010). 'Cardiovascular medicines and primary health care: a regional analysis.' Catalogue No. CVD 48. (AIHW: Canberra)

Bailie RS, Si D, O’Donoghue L, Dowden M (2007) Indigenous health: effective and sustainable health services through continuous quality improvement. The Medical Journal of Australia 186, 525-527.

Bryman A (1995) 'Quantity and quality in social research.' (Routledge: London)

Couzos S, Murray R (2003). 'Aboriginal primary health care: an evidencebased approach.' (Oxford University Press: Melbourne)

Cunningham J, Cass A, Arnold PC (2005) Bridging the treatment gap for Indigenous Australians. The Medical Journal of Australia 182, 505-506.

DiGiacomo ML, Davidson PM, Taylor KP, Smith JS, Dimer LA, Ali MA, Wood MM, Leahy TG, Thompson SC (2010a) Health information system linkage and coordination are critical for increasing access to secondary prevention in Aboriginal health: a qualitative study. Quality in Primary Care 18, 17-26.

DiGiacomo ML, Thompson SC, Smith JS, Taylor KP, Dimer LA, Ali MA, Wood MM, Leahy TG, Davidson PM (2010b) 'I don't know why they don't come': barriers to participation in cardiac rehabilitation. Australian Health Review 34, 452-457. doi:10.1071/AH09803

Dimer L, Dowling T, Jones J, Cheetham C, Thomas T, Smith J, McManus A, Maiorana AJ (2013) Build it and they will come: Outcomes from a successful cardiac rehabilitation program at an Aboriginal Medical Service. Australian Health Review 37, 79-82. doi:10.1071/AH11122

Eckerman A, Dowd T, Chong E, Nixon L, Gray R, Johnson S (eds) (2010) 'Binan Goonj: Bridging Cultures in Aboriginal Health.' (Elsevier Australia: Armidale, NSW)

Foster MM, Mitchell G, Haines T, Tweedy S, Cornwell P, Fleming F (2008) Does enhanced primary care enhance primary care? Policy-induced dilemmas for allied health professionals. The Medical Journal of Australia 188, 29-32.

Halcomb EJ, Davidson PM, Brown N (2010) Uptake of Medicare chronic disease items in Australia by general practice nurses and Aboriginal health workers. Collegian (Royal College of Nursing, Australia) 17, 57-61. doi:10.1016/j.colegn.2010.04.010

Heeley EL, Peiris DP, Patel AA, Cass A, Weekes A, Morgan C, Anderson CS, Chalmers JP (2010) Cardiovascular risk perception and evidence-practice gaps in Australian general practice (the AusHEART study). The Medical Journal of Australia 192, 254-259.

Huffman MD, Galloway JM (2010) Cardiovascular health in Indigenous communities: successful programs. Heart Lung and Circulation 19, 351-360. doi:10.1016/j.hlc.2010.02.013

Katzenellenbogen JM, Sanfilippo FM, Hobbs MST, Briffa TG, Ridout SC, Knuiman MW, Dimer LA, Taylor KL, Thompson PL, Thompson SC (2010) Incidence of and case fatality following acute myocardial infarction in Aboriginal and Non-Aboriginal Western Australians (2000-2004): a linked data study. Heart Lung and Circulation 19, 717-725. doi:10.1016/j.hlc.2010.08.009

Moffatt S, White W, Mackintosh J, Howel D (2006) Using quantitative and qualitative data in health services research - what happens when mixed method findings conflict? BMC Health Services Research 6, 28. doi:10.1186/1472-6963-6-28

National Health and Medical Research Council (2003) Values and ethics: guidelines for ethical conduct in Aboriginal and Torres Strait Islander Health Research. Available at http://www.nhmrc.gov.au/guidelines/ publications/e52 [Verified 14 January 2013] 
National Health and Medical Research Council (2010) The NHMRC road map II: a strategic framework for improving the health of Aboriginal and Torres Strait Islander people through research. NHMRC, Canberra.

One21 seventy (2011) National Centre for Quality Improvement in Indigenous Primary Health Care. Available at http://www.one21seventy.org.au/ [Verified 14 January 2013]

Østbye T, Yarnall KSH, Krause KM, Pollak KI, Gradison M, Michener JL (2005) Is there time for management of patients with chronic diseases in primary care? Annals of Family Medicine 3, 209-214. doi:10.1370/ afm. 310

Pierce D (2009) Identifying and addressing barriers to the use of enhanced primary care plans for chronic disease in rural practices. The Australian Journal of Rural Health 17, 220-221. doi:10.1111/j.1440-1584.2009. 01076.x

Redfern J, Maiorana A, Neubeck L, Clark A, Briffa T (2011) Achieving coordinated secondary prevention of coronary heart disease for all in need (SPAN). International Journal of Cardiology 146, 1-3. doi:10.1016/ j.ijcard.2010.08.046
Schofield D (1999) Ancillary and specialist health services: the relationship between income, user rates and equity of access. The Australian Journal of Social Issues 34, 79-96.

Shepherd F, Battye K, Chalmers E (2003) Improving access to cardiac rehabilitation for remote Indigenous clients. Australian and New Zealand Journal of Public Health 27, 632-636.

Stoneman J, Taylor SJ (2007) Improving access to medicines in urban, regional and rural Aboriginal communities-is expansion of Section 100 the answer? Rural and Remote Health 7, 738.

Thompson SC, Walker AT (2011) Use of modern technology as an aid to medication adherence: an overview. Patient Intelligence 3, 49-55. doi:10. 2147/PI.S8485

Vos T, Barker B, Begg S, Stanley L, Lopez AD (2009) Burden of disease and injury in Aboriginal and Torres Strait Islander Peoples: the Indigenous health gap. International Journal of Epidemiology 38, 470-477. doi:10.1093/ije/dyn240 